\title{
Matrix matching in quantitative bioanalysis by LC-MS/MS a dream or a reality?
}

\author{
Olivier Heudi*,1, Serge Winter ${ }^{1}$ \& Franck Picard ${ }^{1}$ \\ ${ }^{1}$ Novartis Pharma AG, DMPK/Bioanalytics, CH-4056 Basel, Switzerland \\ *Author for correspondence: Tel.: +41795359 611; Olivier.heudi@novartis.com
}
"Ultimately, each study sample should be treated as a unique one. Therefore, is it practical to rely on an exact matrix matching in calibration standards with the samples, when each sample contains different co-eluting compounds at different concentrations and time points?"

First draft submitted: 18 October 2019; Accepted for publication: 7 November 2019; Published online: 19 December 2019

Keywords: internal standard $\bullet$ LC-MS $\bullet$ LC-MS/MS quantitation $\bullet$ matrix effect $\bullet$ validation

\section{LC-MS/MS quantitative analysis \& ESI}

Liquid chromatography coupled with atmospheric pressure ionization tandem MS (LC-MS/MS) is widely used for the quantitative analysis of small molecules in pharmaceutical industry. The high sensitivity and selectivity of LC-MS/MS apparatus have enabled the development of methods with minimum sample preparation, short separation time and detection in ESI mode in an environment where a large amount of endogenous compounds can co-exist with the analyte of interest [1-3]. However, it has been clearly shown that when ESI is used, the intensity of the protonated molecule of organic base decreases significantly in the presence of an increasing amount of another basic compound [1,4]. These co-elute compounds could significantly affect the analyte MS response and the accuracy of the quantitative data.

\section{In sample internal standard monitoring \& in sample calibration curve}

In the US FDA Bioanalytical Method Validation Guidance for Industry [5] and the EMA Guideline on Bioanalytical Method Validation [6], it is stated that calibration standards have to be prepared in the same biological matrix as the samples in the intended study. Moreover, matrix effect and recovery has to be determined in hemolyzed, hyperlipidemic plasma samples in case anticipated in the study [6]. In addition, investigations has to be performed in plasma sample from special populations (renally or hepatically impaired). Another point to consider is the impact of formulation excipients on concentration data obtained on the early time point for samples collected after infusion or of intravenous administration [6]. However, not all these approaches are without their limitations. Most spiked samples fail to mimic the incurred samples as each sample has co-eluting, interfering compounds that are thereby exposed to a different extent of ionization suppression [7]. Ultimately, each study sample should be treated as a unique one. Therefore, is it practical to rely on an exact matrix matching in calibration standards with the samples, when each sample contains different co-eluting compounds at different concentrations and time points? One way to correct for the analyte MS signal suppression or enhancement is to use during the sample analysis an internal standard (IS) - preferably a (stable isotope labeled SIL) analog, which has identical chemical and physical properties to the target analyte [8]. Since it is processed along with the analyte, this IS should compensate for the losses that may occur during the sample preparation. In a recent guidance, the FDA recommends to check the IS responses in each analyzed samples and to further investigate on samples with the IS signals that deviate from the rest of the spiked samples prepared in the same matrix [9]. However, performing these investigations remain a daunting challenge as one can only speculate on the origin of the IS signal variability due the complexity of each plasma sample composition. Hence, it would be unfair to discard sample PK concentrations based on abnormal IS response. In reality, this approach is not in line with attributed function of the IS in the quantitative analysis by LC-MS/MS. Since the IS has the same physical properties to that of the analyte and the IS co-elute, both will experience the same sort of ionization (enhancement or suppression); hence, the analyte to IS peak area (height) 
ratio remains unaffected [10]. In a recent study where $\operatorname{IgG}$ were determined in preclinical species by LC-MS/MS using the bottom-up approach, we have demonstrated that accurate data were obtained when measuring quality control (QC) spiked monkey plasma samples against a calibration curve prepared in rat plasma, despite the IS signal difference observed with the two plasma matrices [11]. This will mean that there is no need to adopt the matrix matching concept and that a generic matrix can be used to generate external calibration curves. Consequently, a unique matrix, human plasma for example, can be considered to analyze samples from other species such as monkey, rat or dog, providing that a proper IS, preferably labeled with ${ }^{13} \mathrm{C}$ or ${ }^{15} \mathrm{~N}$ atoms with identical physical properties (same elution time and ionization pattern) to that of the analyte is used. This approach will avoid the use of multiple matrices and preclude the development of surrogate matrices when handling rare matrices.

In realizing the impracticality of exact matrix matching, Gu et al. [12] recently reported a novel in-sample calibration curve (ISCC) methodology. In this approach, multiple naturally occurring isotopologs of stable isotopically labeled IS spiked in the sample at known concentration is used to build the calibration curve in each sample. Hence, there is no need to use external calibration obtained in a similar matrix and this eliminates the difference between the calibration standard and unknown sample matrices. ISCC will considerably simplify the workout procedure for quantitative analysis by LC-MS/MS, especially for study containing high number of samples and originating from various sources (healthy volunteer, gender, etc.) where assessing the matrix is just not possible."

\section{Conclusion}

The concept one-matrix-fits-all samples (one external calibration curve to measure all incurred sample - with the exception of special cases) led the analyst to carry several matrices and to perform tedious assessments such as matrix effect or to develop surrogate matrices. However, these assessments will not always help understanding the behavior of the analyte in incurred sample matrices. Since we are moving toward the concept of 'personalized incurred samples', it is important to monitor the IS signal during the sample analysis, and if abnormal signal is observed, assess each case without necessarily rejecting the sample concentration found. In fact, in the unknown samples displaying abnormal IS signal, the MS ionization will affect in a similar manner the analyte of interest and its corresponding IS. The advancements made in analytical technology and the development of novel approach such ISCC will help revisiting, simplifying and changing guidelines that will enable analysts to take full advantage of the new developments to cut down the time and cost associated with routine analysis.

\section{Financial \& competing interests disclosure}

The authors have no relevant affiliations or financial involvement with any organization or entity with a financial interest in or financial conflict with the subject matter or materials discussed in the manuscript. This includes employment, consultancies, honoraria, stock ownership or options, expert testimony, grants or patents received or pending, or royalties.

No writing assistance was utilized in the production of this manuscript.

\section{References}

1. Kebarle P, Tang L. From ions in solution to ions in the gas-phase - the mechanism of electrospray mass-spectrometry. Anal. Chem. 65(22), A972-A986 (1993).

2. Matuszewski BK, Constanzer ML, Chavez-Eng CM. Matrix effect in quantitative LC/MS/MS analyses of biological fluids: a method for determination of finasteride in human plasma at picogram per milliliter concentrations. Anal. Chem. 70(5), 882-889 (1998).

3. Matuszewski BK, Constanzer ML, Chavez-Eng CM. Strategies for the assessment of matrix effect in quantitative bioanalytical methods based on HPLC-MS/MS. Anal. Chem. 75(13), 3019-3030 (2003).

4. Gosetti F, Chiuminatto U, Zampieri D, Mazzucco E, Marengo E, Gennaro MC. A new on-line solid phase extraction high performance liquid chromatography tandem mass spectrometry method to study the sun light photodegradation of mono-chloroanilines in river water. J. Chromatogr. A. 1217(20), 3427-3434 (2010).

5. US FDA. Bioanalytical method validation guidance for industry (2018). www.Fda.Gov/Downloads/Drugs/Guidancecomplianceregulatoryinformation/Guidances/Ucm070107.Pdf

6. EMA. Guideline on bioanalytical method validation (2011). www.Ema.Europa.Eu/Docs/En_Gb/Document_Library/Scientific_Guideline/2011/08/Wc500109686.Pdf

7. Hewavitharana AK. Matrix matching in liquid chromatography-mass spectrometry with stable isotope labelled internal standards - is it necessary? J. Chromatogr. A. 1218(2), 359-361 (2011).

8. Van Eeckhaut A, Lanckmans K, Sarre S, Smolders I, Michotte Y. Validation of bioanalytical LC-MS/MS assays: evaluation of matrix effects. J. Chromatogr. B. Analyt. Technol. Biomed. Life Sci. 877(23), 2198-2207 (2009). 
9. US FDA. Evaluation of internal standard responses during chromatographic bioanalysis: questions and answers guidance for industry (2019). www.Fda.Gov/Drugs/Guidancecomplianceregulatoryinformation/Guidances/Default.Htm

10. Liang HR, Foltz RL, Meng M, Bennett P. Ionization enhancement in atmospheric pressure chemical ionization and suppression in electrospray ionization between target drugs and stable-isotope-labeled internal standards in quantitative liquid chromatography/tandem mass spectrometry. Rapid Commun. Mass Spectrom. 17(24), 2815-2821 (2003).

11. Lanshoeft $\mathrm{C}$, Wolf $\mathrm{T}$, Walles $\mathrm{M}$ et al. The flexibility of a generic LC-MS/MS method for the quantitative analysis of therapeutic proteins based on human immunoglobulin G and related constructs in animal studies. J. Pharm. Biomed. Anal. 131, 214-222 (2016).

12. Gu HD, Zhao Y, Demichele M et al. In-sample calibration curve using multiple isotopologue reaction monitoring of a stable isotopically labeled analyte for instant LC-MS/MS bioanalysis and quantitative proteomics. Anal. Chem. 91(3), 2536-2543 (2019). 